- Invited Article -

\title{
State of the Science: Salivary Biomarker Utilization for Stress Research
}

\author{
An, Kyungeh ${ }^{1} \cdot$ Starkweather, Angela ${ }^{2} \cdot$ Sturgill, Jamie L. ${ }^{3} \cdot$ Kao, Hsueh-Fen S. $\cdot$ Salyer, Jeanne ${ }^{2}$ \\ ${ }^{1}$ Associate Professor, School of Nursing, Virginia Commonwealth University, Richmond \\ ${ }^{2}$ Associate Professor, Virginia Commonwealth University, Richmond \\ ${ }^{3}$ Assistant Professor, Virginia Commonwealth University, Richmond \\ ${ }^{4}$ Associate Professor, University of Texas El Paso, Texas, USA
}

\begin{abstract}
Purpose: The use of salivary biomarkers for stress research is increasing based on the convenience of collection, affordability and scientific merit. This short review provides an overview of the state of the science of salivary biomarkers utilized in research related to stress. Methods: An integrative review was conducted. Results: The trend of utilizing salivary biomarkers in stress research was reviewed, specifically, focusing on the use of endocrine and inflammatory biomarkers incorporated in previous stress research. Then, a review of sampling procedures for salivary biomarkers and the analytic methods is provided. Finally, a discussion on the strengths and areas for improvement in the use of salivary biomarkers in stress research is included. Conclusion: Salivary biomarkers as an alternative to blood biomarkers are increasingly being recognized as a legitimate source for analyzing the stress response in humans.
\end{abstract}

Key Words: Stress, Saliva, Biological marker, Cortisol

\section{INTRODUCTION}

A biomarker is defined as "a characteristic that is objectively measured and evaluated as an indicator of normal biological processes, pathogenic processes, or pharmacologic responses to a therapeutic intervention". ${ }^{1)}$ In the last two decades, the term "salivary biomarker" has been used to indicate the biomarkers detected in saliva and related publications have increased dramatically. A literature search on PubMed using the keywords "biomarkers and stress" identified 11,612 results between 2004 and 2014, whereas "salivary biomarkers and stress" identified 302 related publications, a small portion of the body of research devoted to biomarkers and stress. Despite the increased use of saliva for measuring biomarkers, the number of published articles using salivary biomarkers in stress research still accounts for less than $5 \%$ of the total number of publications in this field. This suggests that salivary biomarkers compared to traditional biomarkers are still underutilized in stress research.

The purpose of this paper is to provide an overview of the current state of the science regarding salivary bio- markers for stress research. We will first review the trends of utilizing salivary biomarkers in stress research specifically, we focus on the use of endocrinal and inflammatory biomarkers incorporated in previous stress research. Next, a review of sampling procedures of salivary biomarkers and the analytic methods will be provided for future researchers. Finally, a discussion on the strengths and areas for improvement in the use of salivary biomarkers in stress research is provided.

\section{SUBJECT}

\section{Trends of Utilizing Salivary Biomarkers in Stress Research}

The term "biomarker" dates back as early as $1980 .{ }^{3)}$ The number of laboratories and grants funded specifically designated to conduct research on biomarkers show the rapid growth in the volume of research supported by the National Institute of Health (NIH) of the states and the European Research Council (ERC) or Cancer Research UK in Europe. ${ }^{4-6)}$ Biomarkers have

Corresponding author: An, Kyungeh

School of Nursing, Virginia Commonwealth University, 1100 E. Leigh St. Richmond VA23298, USA.

Tel: +1-804-828-8917, Fax: +1-804-828-7743, E-mail: kan@vcu.edu

투고일 2014년 9월 11일 / 심사완료일 2014년 9월 29일 / 게재확정일 2014년 9월 29일 
been detected predominantly in blood samples and extensively used in clinical trials for their diagnostic value. ${ }^{7,8)}$ With the advancement of biomedical science, assays that are designed specifically for saliva samples have become available, and subsequently, many biomarkers that once required blood samples have been validated in saliva, ${ }^{9,10)}$

Saliva is a clear, slightly acidic $(\mathrm{pH}=6.0 \sim 7.0)$ and protein-rich fluid composed of secretions from major salivary glands: the parotid, submandibular, and sublingual glands. In general., human salivary glands produce about 1 1.5 liter of serous and mucinous saliva daily. ${ }^{11}$ When pathologic health condition arise (e.g., cancer, inflammation), proteins and substances linked to the disease are transferred to the saliva. ${ }^{8,12,13)}$ Increased concentrations of these compounds over time make saliva a diagnostic fluid with numerous advantages over blood. The advantages can be summarized below:

\section{1) Bypassing often painful invasive procedures}

Saliva biomarkers require only non-invasive saliva collection, offers unique opportunities to bypass often painful invasive procedures, such as biopsy and repeated blood draws that add enormous stress to the individuals who already go through unpleasant experience related to their health conditions. Saliva is easy to collect without painful needle sticks, and can be tested in many non-clinical settings for field studies because of portability.

\section{2) Economic consideration}

Salivary biomarkers have economic advantages over blood samples with lower costs to store and process samples, perform tests, and less time for doctor or hospital visits to collect samples, thus improving patient satisfaction.

\section{3) Diagnostic utility}

Use of salivary biomarkers as a diagnostic tool at the point of care has been tested for detection of numerous conditions and diseases, such as acute myocardial infarction, ${ }^{7,14)}$ oral cancer, ${ }^{15-18)}$ pancreatic cancer, ${ }^{19)}$ cardiovascular disease, ${ }^{20-22)}$ human immunodeficiency virus (HIV) status and periodontal disease .7,,11,23) $^{-1}$

\section{4) Scientific merits and convenience for conducting re- search}

Salivary biomarkers offer scientific merits and convenience for conducting research, particularly studies on the physiological mechanisms of the stress response.
Monitoring salivary biomarkers provides a sound, costeffective and relatively simple measure of stress response $e^{24,25)}$ because (a) stability is a great asset of salivary biomarker measurements that allows researchers to monitor biomarkers repeatedly for longitudinal studies, (b) low cost and convenience of the sampling procedure of saliva offers an alternative to blood biomarkers, particularly for large population studies, ${ }^{26)}$ (c) the noninvasive feature of saliva sampling helps stress research in two ways: First, because it is safer than blood draw or biopsy, saliva sampling lessens burden to the participants and encourages individuals who are reluctant to participate due to the anxiety about invasive procedure as supported by subjects' preference of donating saliva to blood or urine samples. ${ }^{27)}$ More importantly, the noninvasive sampling dramatically reduce disturbance related to sampling procedure compared to the conventional blood draw to the research participants, thus minimizing chances of confounding major variables of interest in stress research. With the growing number of studies on salivary biomarkers, the potential for using salivary biomarkers as diagnostic and research tools deserves more attention from the health science and research communities.

\section{Utilization of Salivary Endocrinal and Inflammatory Biomarkers in Stress Research}

Because the ultimate interest of stress research is to understand, predict and modify the impact of stress on human health and behavior, monitoring biomarkers is imperative for understanding the physiologic responses to stress and their impact on human health. Saliva contains a wide range of biomarkers that reflect physiologic responses to perceived stress. Among these, endocrinal and immune markers---such as cortisol and cytokines, respectively---have been extensively used in stress research. For example, the link between psychosocial stress and development of cardiovascular disease has provided a model of stress-health impact that involves the cascading responses of neuro-endocrinal and immunological responses to stress. ${ }^{12,20,24,28-30)}$

The stress response is regulated by two primary neuroendocrine systems: the hypothalamus-pituitaryadrenocortical (HPA) axis and sympathetic adrenomedullary (SAM) systems. ${ }^{31)}$ Psychological stress increases HPA activity and, subsequently, elevates the level of cortisol in circulation. ${ }^{12,24,32,33)}$ On the other hand, the primary mechanism of defense against stressful stimuli is activation of the SAM system, comprising the sympathetic nervous 
system and the adrenal medulla. ${ }^{32)}$ By monitoring cortisol in saliva, we can distinguish individuals who perceive stress whether it is from laboratory-induced experimental stress or real life stress from noise exposure, academic stress, or life difficulties.

Cortisol is the most frequently measured salivary biomarker in stress research. A wide range of salivary biomarkers have been identified and tested for their diagnostic value, and still new biomarkers are being identified and validated. They include dehydroepiandrosteron (DHEA) ${ }^{34)}$ testosterone, and estradiol. Salivary biomarkers also include toxins, metabolites, enzymes ( $\alpha$ amylase, or $\alpha$-amylase), immunoglobulins (IgA), proteins and DNA. ${ }^{35)}$ Saliva contains the insulin sensitivity marker, adiponectin ${ }^{36)}$ cardiac enzymes such as creatinine kinase (CK), myoglobin $(\mathrm{MB})^{37)}$ and creatine phosphokinase $(\mathrm{CPK})^{38)}$ as well as neuroendocrine hormones such as epinephrine, norepinephrine, and DHEA 35. In the following section, we will review three major neuroendocrine markers (cortisol, $\alpha$-amylase, and DHEA) and inflammatory cytokines that are often measured in stress research.

\section{1) Salivary cortisol}

The use of cortisol as a marker for HPA axis activity has been well established in human stress research. ${ }^{12,29,33)}$ Salivary cortisol has a diurnal peak of $13.8 \sim 48.9 \mathrm{nmol} / 1$ compared to blood, 190 690 nmol/1. Since the diurnal rhythm of cortisol release is well known, cortisol is usually measured in a time series, with few exceptions to reflect the awakening stress response, recovery from awakening, as well as its changes throughout the day until bedtime. ${ }^{12,39)}$ Normal salivary cortisol level varies depending on the studies: $0.67 \pm 0.12$ to $15.5 \pm 0.8 \mathrm{nmol} / \mathrm{L}$ (range 10.2 27.3) at 8:00 $\mathrm{AM}^{33)}$ The most frequently used protocol is the one described by Pariante et al., $(2004)^{40)}$ that used the total salivary cortisol output, calculated as the area under the curve (AUC) during the day (MN, $8 \mathrm{AM}$, and $8 \mathrm{PM}$ ) and the AUC of the increase (AUCi) of cortisol level after awakening (from 0 to 15, 30, 60 minutes after awakening). The amount of bioavailable cortisol in saliva assessed by the immune assays reflect the HPA axis activity which is of major interest in both research and clinical diagnosis. ${ }^{41)}$ Also, besides immunoassay, ultra-sensitive nano-tube immunosensor is available for a rapid measurement of salivary cortisol. $^{14)}$

\section{2) Salivary $\alpha(\alpha)$-amylase}

The salivary enzyme $\alpha$-amylase is secreted by the pa- rotid gland. Properties of $\alpha$-amylase have been extensively studied in the last decade. While cortisol reflects the HPA axis activity, $\alpha$-amylase has been used as a novel biomarker of SAM activity. ${ }^{25,42)}$ The potential of salivary $\alpha$-amylase as a salivary biomarker of adrenergic activity is of interest because it allows the parallel investigation of the two major neuroendocrine response systems---HPA and SAM axis---using non-invasive salivary samples. Salivary $\alpha$-amylase levels have been shown to increase in response to both physical and psychological stressors, and offers a non-invasive measure for research on stress responses. ${ }^{42,43)}$ Secretion of $\alpha$ -amylase is increased by acute experimental stressors, but is reduced by chronic stress. $\alpha$-amylase has an endogenous diurnal rhythm like cortisol, thus it may not be reliable marker of catecholamine levels. Another asset of saliva $\alpha$-amylase is its stability. $\alpha$-amylaseactivity is stable over 21 days up to $37^{\circ} \mathrm{C}$, and in the absence of bacterial contamination, bacterial growth is limited. These properties make $\alpha$-amylase activity useful as a biomarker of stress-related autonomic activity that complements plasma catecholamine and cardiac responses to psychosocial stress. ${ }^{25)}$ Stress-related increases in salivary $\alpha$-amylase can be inhibited by administration of the commonly used adrenergic blocker, propranolol, ${ }^{43}$ but the $\beta$-adrenergic agonist stimulates salivary $\alpha$ -amylase release. ${ }^{44)}$ These properties provide investigators with an important methodological caution to avoid those who use prescription medications for angina or high blood pressure that contain a $\beta$-blocker or similar actions in biobehavioral research using salivary $\alpha$-amylase measurements.

The pattern of stress-related change in salivary cortisol and $\alpha$-amylase levels are different from each other. ${ }^{12,45)}$ Both salivary analytics increase in response to psychosocial stress, however, the level of salivary $\alpha$-amylase reaches the peak faster, then, returns to the prestress level faster than the level of salivary cortisol. This difference is consistent with the physiological differences of the sympathetic nervous system (SNS) response (quicker) and HPA (slower) response to stress. The differences may be due to the higher sensitivity to stress in SNS than in HPA axis. ${ }^{33)}$ While cortisol and $\alpha$-amylase reflect different stress mechanisms, they coordinate the stress response to influence cardiovascular health outcomes.

\section{3) Dehydroepiandrosterone (DHEA)}

DHEA is a steroid hormone that is produced in the adrenal cortex and can be found in saliva. DHEA has 
shown inverse correlation with cortisol levels, indicating protective effects against stress. ${ }^{34,35)}$ Shirotsuki (2009) reported that complete HPA axis reactivity to acute psychosocial stressors is blunted in anxious individuals as evidenced by a lower cortisol to DHEA ratio in response to psychosocial stress. This finding suggests that salivary level of DHEA, in addition to cortisol, provides a valuable tool, therefore, should be should be considered when investigating biomarkers of stress in this population. ${ }^{34)}$

\section{4) Inflammatory markers}

A wide range of inflammatory biomarkers hasbeen used for stress research that include interleukin 1 (IL-1), IL-6, C-reactive protein (CRP), and tumor necrosis factor$\alpha($ TNF- $\alpha){ }^{15,46)}$ Inflammatory biomarkers have contributed to advancing our understanding of the role of inflammation in stress physiology. ${ }^{12,30)}$ The HPA axis and autonomic nervous system responses to stress manifest in lowering immune function as shown by numerous studies for the last three decades. Chronic psychosocial stress, such as job strain, low socioeconomic status, and caregiver stress are related to the impairments of immune function, ${ }^{12,30)}$ which are linked to the development of health conditions such as atherosclerosis, ${ }^{21,28,30,47)}$ and cancer. ${ }^{15,16)}$ Salivary immunoglobulin A (IgA) and lysozyme were inversely correlated with self-reported level of stress, suggesting their utility aspotential stress biomarkers. ${ }^{35)}$ Studies to identify and validate new salivary biomarkers for stress research is ongoing.

\section{Saliva Sample Collection, Storage, Centrifugation and Time of Measurements}

Methods for saliva collection have significantly advanced over the last decade, leading to standard and reliable methods, with devices and techniques that produce consistent salivary samples that provide the most accurate results. $^{10,13)}$

For salivary sample collection, two standard methods have been used: cotton roll and passive drool. In a review of prior studies incorporating salivary biomarkers, some studies describe whether they used cotton roll or passive drool, ${ }^{10,22)}$ but most did not specify the method used to collect saliva samples. Saliva samples must be collected in a protease free tubes to optimize stability for a long term storage. After collecting saliva samples, storage conditions and the centrifugation process before assay are similar yet inconsistently reported across studies. Saliva samples are stored in $-18,-20$, or $-80^{\circ} \mathrm{C}$ freezer until assay. ${ }^{47)}$ Centrifugation was at 3,000 3,500 rpm and the duration of centrifugation varied between 3 minutes $^{47)}$ and 10 minutes. $^{20)}$

Some studies described sampling time to capture the peak level of cortisol or when the diurnal rhythm was considered to affect the biomarkers of interest. ${ }^{48)}$ For example, the level of cortisol was measured between directly after awakening and 20 or 30 minutes after awakening to capture the peak level. ${ }^{20,47)}$ then, saliva samples were collected several times during the day until evening and at bedtime ${ }^{20,21,47)}$ to investigate the diurnal rhythm of cortisol. When urine samples were collected along with saliva, similar time intervals were used for levels of cortisol as well as epinephrine and norepinephrine. ${ }^{28)}$ When the time series was not chosen, sampling time for saliva cortisol was usually in the morning. ${ }^{49)}$

\section{Issues related to Use of Salivary Biomarkers}

Much attention has been paid to improving the measurement of salivary biomarkers, mainly due to its nature of non-invasive sampling that gives much convenience to both researchers and participants. ${ }^{27)}$ We will discuss some concerns and its remediation below

\section{1) Reaching optimum reliability and validity}

For optimum reliability and validity, saliva sampling, storage and analysis have to strictly follow standard procedures (see description above). In studies that require research participants to collect saliva by themselves, detailed guidance should be provided with demonstration and even with practice, if necessary. To conquer this concern, our recent study has been successful in providing guidance by using a video clip that is stored on the participant's mobile device which provides details for the sampling procedures of saliva. ${ }^{50)}$

\section{2) Difficulty in some populations}

In very young children, older or frail populations, collecting saliva can be unexpectedly difficult, time consuming, and may not yield a sufficient volume of specimen for assay. Granger and colleagues $(2007)^{10)}$ provided a list of these pitfalls and made suggestions for future researchers. Challenges in saliva collection from full-term and preterm newborns, and infants less than 3 months of age has been well documented. ${ }^{51)}$ More time is needed to collect sufficient volume of saliva for assays because newborn infant's parotid glands have low fluid production rates compared to older children and adults. On the opposite end, collecting saliva samples from the 
oldest and most frail elderly participants can also be difficult because of their chronic health conditions, cognitive impairment, immobility, and fatigue. More importantly, poor hydration practices and the impact of multiple medications can make collecting sufficient saliva volume challenging and may take extra time in geriatric populations. ${ }^{52)}$ Therefore, checking the list of medications and their iatrogenic effects on restriction of saliva flow may be necessary for studies with oldest old population.

\section{3) Low concentration of analytes in saliva}

Although saliva contains as diverse a number of diagnostic components as blood samples, there have been concerns that the low concentration compared with levels in the blood may prevent salivary diagnostics from being clinically useful. However, with the development of new and highly sensitive techniques (e.g., molecular diagnostics, nanotechnology), the low concentration of analytes in saliva is no longer a limitation. ${ }^{9,14,23,53,54)}$ To date, molecular and proteomic technology yield advanced insight into the characteristics of salivary proteomes and provide strong evidence supporting the use of saliva as a diagnostic tool. . $^{9,17,23,53-56)}$

In sum, biomarker research is advancing the ability to profile a large number of analytes in a single assay using high throughput genomics, proteomics, ${ }^{17)}$ and other 'omics' platforms. Together with the pending prospect of rapid expansion of whole exome and whole genome sequencing for clinical use, new biomarkers will be identified and validated and the technical and logistical complexity of biomarker validation will be increasing. As Poste (2012) proposed, connecting these new technologies and improving productivity in biomarker validation will be the key for clinically meaningful outcomes out of this biomarker enterprise. ${ }^{4)}$ Likewise, new salivary biomarkers should be identified and validated for improved patient management and clinical outcomes for those who suffer diverse forms of stress-related health issues.

\section{CONCLUSION}

The use of salivary biomarkers for stress research is increasing based on the convenience of collection, affordability and scientific merit. Instead of considering salivary biomarkers as an alternative to blood, it is increasingly being recognized as a legitimate source for analyzing the stress response in humans.

\section{REFERENCES}

1. Atkinson AJ, Colburn WA, DeGruttola VJ, DeMets DL, Downing GJ, Hoth DF, et al. Woodcock J, Zeger SL. Biomarker definition working group. Biomarkers and surrogate endpoints: preferred definitions and conceptual framework. Clin Pharmacol Ther. 2001;69(3):89-95.

http://dx.doi.org/10.1067/mcp.2001.113989.

2. National Center for Biotechnology Information. Available from: http://www.ncbi.nlm.nih.gov/pubmed/?term=salivary+biom arkers+and+stress. Accessed 08/24/2014.

3. Aronson JK. Biomarkers and surrogate endpoints. Br J Clin Pharmacol. 2005;59(5):491-94.

http://dx.doi.org/10.1111/j.1365-2125.2005.02435.x.

4. Poste G. Biospecimens, biomarkers, and burgeoning data: the imperative for more rigorous research standards. Trend Mol Med. 2012;18(12):717-22.

http://dx.doi.org/10.1016/j.molmed.2012.09.003

5. National Institute of Environmental Health Sciences. Available from: http://www.niehs.nih.gov/health/topics/science/bio markers. Accessed 2014.

6. Cancer Research UK. Available from: http://www.cancerre searchuk.org/funding-for-researchers/our-funding-schem es/biomarker-project-awards. Accessed 8/31/2014

7. Christodoulides N, Florian PN, Miller CS, Ebersole JL, Mohanty S, Dharshan P, et al. Lab-on-a-chip methods for point-ofcare measurements of salivary biomarkers of periodontitis. Ann N Y Acad Sci. 2007 Mar;1098:411-28.

8. Srinivas PR, Kramer BS, Srivastava S. Trends in biomarker research for cancer detection. Lancet Oncol, 2001;2(11):698-704. http://dx.doi.org/10.1016/S1470-2045(01)00560-5

9. Lee Y-H, Wong DT. Saliva: An emerging biofluid for early detection of diseases. Am J Dent. 2009;22(4):241-8.

10. Granger DA, Kivlighan KT, Fortunato C, Harmon AG, Hibel LC, Schwartz EB, et al. Integration of salivary biomarkers into developmental and behaviorally-oriented research: Problems and solutions for collecting specimens. Physiol Behav. 2007; 92(4):583-90.

11. Wong DTW. Salivary Diagnostics: Scientific and Clinical Frontiers. Advances in Dental Research. 2011;23(4):350-2.

12. Cohen S, Schwartz JE, Epel E, Kirschbaum C, Sidney S, Seeman T. Socioeconomic status, race, and diurnal cortisol decline in the coronary artery risk development in young adults (CARDIA) study. Psychosomatic Med. 2006;68(1):41-50. http://dx.doi.org/10.1097/01.psy.0000195967.51768.ea

13. Salimetrics. Available from: https://www.salimetrics.com/col lection-systems. Accessed 8/31/2014.

14. Floriano PN, Christodoulides N, Miller CS, Ebersole JL, Spertus J, Rose BG, et al. Use of saliva-based nano-biochip tests for acute myocardial infarction at the point of care: a feasi- 
bility study. Clin Chem. 2009;55(8):1530-8.

http://dx.doi.org/10.1373/clinchem.2008.117713 Epub 2009 Jun 25.

15. Brailo V, Vucicevic-Boras V, Lukac J, Biocina-Lukenda D, Zilic-Alajbeg I, Milenovic A, et al. Salivary and serum interleukin 1 beta, interleukin 6 and tumor necrosis factor $\alpha$ in patients with leukoplakia and oral cancer. Medicina Oral, Patología Oraly Cirugía Buccal. 2012;17(1):e10.

16. Goulart BHL, Clark JW, Pien HH, Roberts TG, Finkelstein SN, Chabner BA. Trends in the use and role of biomarkers in phase I oncology trials. Clin Cancer Res. 2007;13:6719. http://dx.doi.org/10.1158/1078-0432.CCR-06-2860

17. Hu S, Arellano M, Boontheung P, Wang J, Zhou H, Jiang J, et al. Salivary proteomics for oral cancer biomarker discovery. Clin Cancer Res. 2008;14(19):6246-52.

18. Park NJ, Zhou H, Elashoff D, Henson BS, Kastratovic DA, Abemayor E, et al. Characterization, and Clinical Utility for Oral Cancer Detection. Clin Cancer Res. 2009;15:5473-7. http://dx.doi.org/10.1158/1078-0432.CCR-09-0736

19. Zhang L, Farrell JJ, Zhou H, Elashoff D, Akin D, Park NH, et al. Salivary transcriptomic biomarkers for detection of resectable pancreatic cancer. Gastroenterology. 2010;138(3):94957, e1-7. http://dx.doi.org/10.1053/j.gastro.2009.11.010. Epub 2009.

20. Dekker MJ, Koper JW, van Aken MO, Pols HA, Hofman A, de Jong FH, et al. Salivary cortisol is related to atherosclerosis of carotid arteries. J Clin Endocrinol Metabolism. 2008;93(10): 3741-7.

http://dx.doi.org/10.1210/jc.2008-0496; 10.1210/jc.2008-0496

21. DeSantis AS, DiezRoux AV, Hajat A, Aiello AE, Golden SH, Jenny NS, et al. Associations of salivary cortisol levels with inflammatory markers: The multi-ethnic study of atherosclerosis. Psychoneuroendocrinology. 2012;37(7):1009-18. http://dx.doi.org/10.1016/j.psyneuen.2011.11.009;10.1016/j. psyneuen.2011.11.009

22. Foley JD III, Sneed JD, Steinhubl SR. Oral fluids that detect cardiovascular disease biomarkers. Oral Surgery Oral Medi Oral Pathol Oral Radiol. 2012;114(2):207-14.

23. Herr AE, Hatch AV, Throckmorton DJ, Tran HM, Brennan JS, Giannobile WV, Singh AK. Microfluidic immunoassays as rapid saliva-based clinical diagnostics. Proc Natl Acad Sci USA. 2007; 104(13):5268-73. Epub 2007 Mar 20.

24. Hellhammer DH, Wüst S, Kudielka BM. Salivary cortisol as a biomarker in stress research. Psychoneuroendocrinology. 2009;34(2):163-71.

http://dx.doi.org/10.1016/j.psyneuen.2008.10.026. Epub 2008 Dec 18

25. De Caro JA. Methodological considerations in the use of salivary $\alpha$-amylase as a stress marker in field research. Am J Hum Biol. 2008;20(5):617-9.
26. Adam EK, Kumari M. Assessing salivary cortisol in large-scale, epidemiological research. Psychoneuroendocrinology. 2009; 34(10):1423-36.

http://dx.doi.org/10.1016/j.psyneuen.2009.06.011

27. Dhima M, Salinas TJ, Wermers RA, Weaver AL, Koka S. Patients preference(Preference changes of adult outpatients for giving saliva, urine and blood for clinical testing after actual sample collection. J Prosthodont Res. 2013 January;57(1):51-6. http://dx.doi.org/10.1016/j.jpor.2012.09.004

28. Eller NH, Netterstrom B, Allerup P. Progression in intima media thickness--the significance of hormonal biomarkers of chronic stress. Psychoneuroendocrinology. 2005;30(8):715-23. http://dx.doi.org/10.1016/j.psyneuen.2005.01.005

29. Hajat A, Diez-Roux A, Franklin TG, Seeman T, Shrager S, Ranjit N, et al. Socioeconomic and race/ethnic differences in daily salivary cortisol profiles: The Multi-Ethnic Study of Atherosclerosis. Psychoneuroendocrinology. 2010;35(6):932-43. http://dx.doi.org/10.1016/j.psyneuen.2009.12.009.

30. Karmack T. Psychosocial stress and cardiovascular disease: An exposure science perspective Psychological Science Agenda I April 2012.

31. Takai N, Yamaguchi M, Aragaki T, Eto K, Uchihashi K, Nishikawa Y. Gender-specific differences in salivary biomarker responses to acute psychological stress. Ann N Y Acad Sci. 2007;1098:510-5.

32. Cohen S, Kessler RC, Underwood GL. Strategies for measuring stress in studies of psychiatric and physical disorders. In: Cohen S, Kessler RC, \& Underwood Gordon L. editor. Measuring Stress: A guide for health and social scientists. New York: Oxford University Press; 1995.

33. Laudat MH, Cerdas S, Fournier C, Guiban D, Guilhaume B, Luton JP. Salivary cortisol measurement: a practical approach to assess pituitary-adrenal function. J Clin Endocrinol Metab. 1988;66(2):343-8.

34. Shirotsuki K, Izawa S, Sugaya N, Yamada KC, Ogawa N, Ouchi Y, et al. Salivary cortisol and DHEA reactivity to psychosocial stress in socially anxious males. Int J Psychophysiol. 2009;72(2):198-203.

35. Vitorino R, Lobo MJC, Ferrer-Correira AJ, Dubin JR, Tomer $\mathrm{KB}$, Domingues PM, et al. Identification of human whole saliva protein components using proteomics. Proteomics. 2004;4 (4):1109-15

36. Weber-Hamann B, Kratzsch J, Kopf D, Lederbogen F, Gilles $\mathrm{M}$, Heuser I, et al. Resistin and adiponectin in major depression: The association with free cortisol and effects of antidepressant treatment. J Psychiatr Res. 2007;41(3-4):344-50. http://dx.doi.org/10.1016/j.jpsychires.2006.01.002

37. Mirzaii-Dizgah I, Hejazi SF, Riahi E, Salehi MM. Saliva-based creatine kinase $\mathrm{MB}$ measurement as a potential point-of-care testing for detection of myocardial infarction. Clin Oral Inves- 
tig. 2012;16(3):775-9.

38. Mirzaii-Dizgah I, Jafari-Sabet M. Unstimulated whole saliva creatine phosphokinase in acute myocardial infarction. Oral Dis. 2011;17(6):597-600

http://dx.doi.org/10.1111/j.1601-0825.2011.01817.x; 10.1111 / j.1601-0825.2011.01817.x

39. Matthews K, Schwartz J, Cohen S, Seeman T. Diurnal cortisol decline is related to coronary calcification: CARDIA study. Psychosomatic Med. 2006;68(5):657-61. http://dx.doi.org/10.1097/01.psy.0000244071.42939.0e

40. Pariante CM, Papadopoulos AS, Poon L, Cleare AJ, Checkley SA, English J, et al. Four days of citalopram increase suppression of cortisol secretion by prednisolone in healthy volunteers. Psychopharmacology. 2004;177(1-2):200-6.

41. Tlili C, Myung NV, Shetty V, Mulchandani A. Label-free, chemiresistor immunosensor for stress biomarker cortisol in saliva. Biosens Bioelectron. 2011;26(11):4382-6.

42. Nater UM, Rohleder N. Salivary alpha amylase as a non-invasive biomarker for the sympathetic nervous system: current state of research. Psychoneuroendocrinology. 2009;34(4):48696.

43. van Stegeren A, Rohleder N, Everaerd W, Wolf OT. Salivary alpha amylase as marker for adrenergic activity during stress: effect of betablockade. Psychoneuroendocrinology. 2006;31 (1):137-41.

44. Rohleder N, Nater UM, Wolf JM, Ehlert U, Kirschbaum C. Psychosocial stress-induced activation of salivary alpha-amylase: an indicator of sympathetic activity? Ann N Y Acad Sci. 2004;032:258-63.

45. de Rooij SR. Blunted cardiovascular and cortisol reactivity to acute psychological stress: a summary of results from the Dutch Famine Birth Cohort Study. Int J Psychophysiol. 2013; 90(1):21-7. http://dx.doi.org/10.1016/j.ijpsycho.2012.09.011

46. SahebJamee M, Eslami M, Atarbashi Moghadam F, Sarafnejad A. Salivary concentration of TNF alpha, IL1 alpha, IL6, and IL8 in oral squamous cell carcinoma. Medicina Oral, Patología Oral Cirugía Bucal. 2008;13(5):E292-5.

47. DeSantis AS, DiezRoux AV, Hajat A, Golden SH, Jenny NS, Sanchez BN, et al. Associations of salivary cortisol levels with metabolic syndrome and its components: The multi-ethnic study of atherosclerosis. J Clin Endocrinol Metab. 2011;96 (11):3483-92.

http://dx.doi.org/10.1210/jc.2011-0483; 10.1210/jc.2011-0483

48. Hibel LC, Granger DA, Cicchetti D, Rogosch F. Salivary Biomarker Levels and Diurnal Variation: Associations With Medications Prescribed to Control Children's Problem Behavior. Child Dev. 2007 May/June;78(3):927-37.

http://dx.doi.org/10.1111/j.1467-8624.2007.01041.x

49. Do DP, Diez Roux AV, Hajat A, Auchincloss AH, Merkin SS, Ranjit N, et al. Circadian rhythm of cortisol and neighborhood characteristics in a population-based sample: The multi-ethnic study of atherosclerosis. Health Place. 2011;17(2):625-32. http://dx.doi.org/10.1016/j.healthplace.2010.12.019

50. An K, Salyer J, Kao HF. Psychological strains, salivary biomarkers, and risks for coronary heart disease among hurricane survivors. Biol Res Nurs. 2014 Sep 18. pii: 109980041455 1164. [Epub ahead of print]

51. Bettendorf M, Albers N, Bauer J, Heinrich UE, Linderkamp O, Maser-Gluth C. Longitudinal evaluation of salivary cortisol levels in full-term and preterm neonates. Horm Res. 1998;50: $303-8$

52. Hodgson N, Freedman VA, Granger DA, Erro A. Biobehavioral correlates of relocation in the frail elderly: salivary cortisol, affect, and cognitive function. J Am Geriatr Soc. 2004;52:185662.

53. Schrohl AS, Würtz S, Kohn E, Banks RE, Nielsen HJ, Sweep FC, et al. Banking of biological fluids for studies of diseaseassociated protein biomarkers. Mol Cell Proteomics. 2008;10: 2061-6. http://dx.doi.org/10.1074/mcp.R800010-MCP200

54. Li Y, Zhou X, St John MAR, Wong DT. RNA profiling of cellfree saliva using microarray technology. J Dent Res. 2004;83: 199-203.

55. National Institute of Dental and Craniofacial Research (NIDCR). Salivary Diagnostics, NIH fact sheets. Available from: http://report.nih.gov/nihfactsheets/ViewFactSheet.aspx?csid $=65$ Updated 2010. Accessed on 8/28/14

56. Yoshizawa JM, Schafer CA, Schafer JJ, Paster BJ, Wong DTW. Salivary biomarkers: toward future clinical and diagnostic utilities. Clin Microbiol Rev. 2013;26(4):781-91. http://dx.doi.org/10.1128/CMR.00021-13 